RECOLECCIÓN DE ELEMENTOS PARA LA CARACTERIZACIÓN DE LA VULNERABILIDAD TERRITORIAL EN LA CUENCA MEDIO-ALTA DEL RÍO NAGUA, REPÚBLICA DOMINICANA

Collection of elements to characterize land vulnerability in the medium-upper basin of Nagua River, Dominican Republic

\title{
Michela Izzo
}

Resumen: El presente artículo contiene los resultados de una investigación orientada a sentar las bases para la elaboración de modelos ambientales de cuencas hidrográficas, con enfoque en la identificación de factores clave para la definición de su vulnerabilidad frente a diferentes amenazas.

El análisis se articuló según cuatro componentes: socio-económico, basado en el uso del Enfoque de Medios de Vida Sostenibles (EMVS) y el Marco de Capitales de la Comunidad (MCC); meteoclimático, basado en el levantamiento in situ de variables termopluviométricas, así como en el procesamiento de datos climatológicos; forestal, basado en el establecimiento de una parcela de medición permanente; evolución de cobertura, basada en el uso de imágenes satelitales de diferente período.

Los resultados obtenidos muestran diferentes niveles de interacción de la población residente con su territorio. De manera específica, puede observarse una progresiva urbanización de la población pasando de áreas de cuenca alta a áreas de cuenca baja, donde se verifica un incremento de las fuentes de ingreso y, a la vez, del 
individualismo. El EMVS y el MCC han resultado herramientas adecuadas para identificar los factores que pueden determinar el éxito de iniciativas de desarrollo de la comunidad, así como los puntos críticos a tomar en cuenta en estrategias de acompañamiento.

Los datos de lluvia levantados en el período mayo-noviembre de 2015 evidencian que la zona está experimentando un profundo déficit pluviométrico (más de $500 \mathrm{~mm}$ en siete meses), efecto de la influencia de El Niño en el área del Caribe. El establecimiento de un punto de medición en la cuenca es un paso clave para la modelación hidrológica y ambiental de la misma.

Los resultados obtenidos constituyen una base importante para avanzar en la caracterización de la vulnerabilidad de las cuencas hidrográficas, contribuyendo a llenar un vacío de conocimiento existente en el país.

Palabras clave: Vuelta Larga, análisis de vulnerabilidad, parcela forestal de medición permanente, cuenca-medio alta, análisis diacrónico.

Abstract: In this article we present the results of a research oriented to set the bases for the elaboration of environmental models of hydrographic basins, with special focus on the identification of key factors for the definition of their vulnerability to different threats.

The analysis was structured into four components: socioeconomic, based on the use of the Sustainable Livelihoods Approach (SLA) and the Community Capitals Framework (CCF); meteo-climatic, based on in situ measurement of temperature and rain, and on processing climatic data; forestry, based on the installation of a permanent parcel; land cover evolution, based on the use of satellite images of different periods. 
Recolección de elementos para la caracterización de la vulnerabilidad territorial en la cuenca medio-alta del Río Nagua, República Dominicana

The obtained results show different levels of interaction of resident population with its territory. Specifically, a progressive urbanization can be observed proceeding from upper to lower basin areas, with an increase of sources of incomes, as well as of individualism. The SLA and CCF show to be an appropriate tool to identify those factors which can determine the success of community development initiatives, as well as critical points for a good strategy of accompaniment.

Rain data which were registered during May to November 2015 show that the zone is experimenting a deep rain deficit (more than $500 \mathrm{~mm}$ in seven months), as an effect of the influence of the El Niño in the Caribbean region. A weather station in the basin is a key step in the process of its hydrological and environmental modeling.

Tree cover is very good in the Vuelta Larga basin, where a substantial stability can be observed in a period of 12 years (2000-2012), with a very small reduction $(-2.6 \%)$, prevalently linked to agriculture of minor crops and the renovation of cocoa plantation.

The obtained results are an important base to progress in vulnerability characterization of hydrographic basins, contributing to fill the knowledge gap, which exists in the country.

Keywords: Vuelta Larga, vulnerability analysis, permanent parcel, account to medium high, diachronic analysis. 


\section{Antecedentes}

El territorio de la República Dominicana cuenta con una enorme diversidad de ambientes, debido a su típica orografía. La estructura y orientación de las cadenas de montaña del país constituyen una barrera frente a los vientos Alisios, principales transportadores de humedad. Esto determina una profunda variación de los regímenes de precipitación en el territorio nacional, generando climas que varían del extremadamente árido al tropical perhúmedo (Izzo et al., 2010). Dicha diversidad climática se traduce en una muy amplia variedad de ambientes, que permite catalogarla como el país "entre el bosque pluvial y el desierto" (Bolay, 1997), distinguiéndose como uno de los botspots de biodiversidad del planeta (Huggins et al., 2007).

A la riqueza se suma la presencia de ambientes frágiles, así como de numerosos y difusos fenómenos de degradación de tierra, características que, en su conjunto, hacen que el territorio dominicano presente elevados niveles de vulnerabilidad frente a eventos meteo-climáticos, incluyendo el cambio climático (SEMARENA, 2004; IPCC, 2007; SEMARENA, 2009; Izzo et al., 2012; IPCC, 2013). Investigaciones recientes llevadas a cabo en el país han evidenciado la existencia de modificaciones significativas de la temperatura y los patrones de lluvia (Izzo, 2011; Pérez \& Jury, 2012): en los últimos setenta años las temperaturas mínima y máxima promedio anuales han venido creciendo de $3.0 \pm 0.5^{\circ} \mathrm{C}$ y $1.8 \pm$ $0.4^{\circ} \mathrm{C}$ respectivamente, mientras que se ha venido observando una reducción significativa de las precipitaciones especialmente en las zonas sotavento de las principales cadenas de montaña, un incremento de la erosividad de la lluvia y un aumento de la evapotranspiración (Izzo, 2011). Además, un estudio conducido a escala nacional para evaluar la vulnerabilidad del territorio dominicano frente al cambio climático (Izzo et al., 2012) destaca que trece provincias del país (alrededor del $40 \%$ del territorio nacional) presentan una vulnerabilidad de alta a muy alta frente a eventos 
Recolección de elementos para la caracterización de la vulnerabilidad territorial en la cuenca medio-alta del Río Nagua, República Dominicana

climáticos. Profundizaciones conducidas en el sector agrícola, evidencian que cultivos clave para la economía de la República Dominicana, como son el café y el banano, presentan numerosos elementos que los hacen extremadamente vulnerables frente al cambio climático (Izzo, 2012a; 2012b). Un análisis de sensibilidad a la desertificación evidencia que el $48 \%$ del territorio nacional es críticamente sensible a dicho fenómeno, siendo el uso intenso e inapropiado de los recursos naturales un factor clave en aumentar la sensibilidad frente al mismo (Izzo et al., 2013).

Uno de los hallazgos de los estudios citados es que el factor humano ejerce un rol determinante en condicionar, positivamente o negativamente, el estado del territorio y, en general, su vulnerabilidad frente a diferentes amenazas. Un ejemplo de acciones que reducen la degradación del territorio y favorecen el establecimiento de modelos sostenibles de aprovechamiento de los recursos naturales es constituido por los sistemas microhidroeléctricos comunitarios, donde grupos locales organizados, bajo principios de fortalecimiento de las capacidades y empoderamiento local, asumen el desafío de la construcción de la microhidroeléctrica y, al terminarla, establecen un sistema autónomo de manejo de la misma (PPS-SGP, 2015).

Los territorios habitados por las comunidades empeñadas en el desarrollo de este proceso representan el espacio ideal para realizar estudios que permitan relacionar factores físicos y socio-económicos e identificar las variables clave para la caracterización de las cuencas hidrográficas en términos de vulnerabilidad frente a determinadas amenazas.

\section{Problema de la investigación}

En tema de definición de la vulnerabilidad del territorio, un aspecto de importancia significativa es la identificación de elementos clave, prioritarios a la hora de determinar el estado de degradación de un ambiente a diferente escala. Específicamente, constituye un reto 
caracterizar la relación existente entre variables físicas y socioeconómicas, identificando las correlaciones más significativas para la descripción y evaluación de la vulnerabilidad territorial frente a eventos específicos. En este esfuerzo, las cuencas hidrográficas son un espacio físico bien definido donde puedan establecerse metodologías más apropiadas y cuantitativas para el análisis de la vulnerabilidad del territorio frente al cambio climático y otras amenazas.

Vuelta Larga es un paraje en el municipio de El Factor (provincia María Trinidad Sánchez), a unos 21 kilómetros del pueblo de Nagua y 65 kilómetros de San Francisco de Macorís, en la vertiente meridional de la Loma Guaconejo, en la porción más oriental de la Cordillera Septentrional. La población local, constituida por 90 familias cuya economía está basada prevalentemente en los cultivos del cacao y del coco, no cuenta con el acceso al servicio eléctrico. Actualmente está empeñada en el proceso de construcción de un sistema microhidroeléctrico comunitario de $21 \mathrm{~kW}$ de potencia, el cual brindará electricidad a los moradores de la comunidad, a partir del aprovechamiento de las aguas del Arroyo Vuelta Larga, afluente de izquierda del Río Nagua (PPS-SGP, 2015).

A pesar de ser una comunidad relativamente aislada y de difícil acceso, Vuelta Larga cuenta con una población juvenil significativa: el 33\% de las personas que viven en la comunidad tiene menos de 30 años y cuenta con un buen nivel de instrucción. Con el objetivo de favorecer el establecimiento de condiciones que generen alternativas formativas y de ingreso, la comunidad, con especial involucramiento de los jóvenes de la zona, ha empezado un proceso de "universidad del campo", cuyo objetivo es acercar la universidad a la realidad rural, favoreciendo la formación académica de los jóvenes, y a la vez aprovechar un espacio experimental único donde hacer investigación en diferentes temas, mediante el involucramiento y la participación activa de la población local. 
Recolección de elementos para la caracterización de la vulnerabilidad territorial en la cuenca medio-alta del Río Nagua, República Dominicana

Este contexto constituye el ambiente ideal donde poder llevar a cabo un proceso de estudio de cuencas hidrográficas, orientado a la identificación de factores que permitan definir el estado de vulnerabilidad de las mismas, contribuir a llenar el vacío de información y datos actualmente existentes en el país, y proporcionar elementos importantes para la elaboración de modelos ambientales a diferente escala.

\section{Materiales y métodos}

La investigación estuvo estructurada en cuatro ejes principales.

\subsection{Análisis socio-económico-ambiental}

El diagnóstico participativo se realizó en las comunidades de El Papayo y Vuelta Larga, en la cuenca del Río Nagua, provincia María Trinidad Sánchez.

Se usaron como marco metodológico y conceptual el Enfoque de Medios de Vida Sostenibles (EMVS) y el Marco de los Capitales de la Comunidad (MCC). A través del EMVS se trabaja con la gente, estudiando los diferentes capitales de las comunidades, su potencial y vulnerabilidad y la manera como las comunidades los aprovechan en sus estrategias de vida (Carney, 1999). Este enfoque ha sido utilizado por diversas organizaciones en variados sectores y escenarios, y su interpretación varía en cada uno de ellos, siendo que algunas entidades definen sus políticas de intervención según el EMVS mientras que otras lo han adoptado más someramente para mejorar su programación y marco analítico. Por la naturaleza participativa de su implementación, el EMVS ha permitido introducir mejor los temas de género, poder, derechos y mercados, así como complementar los marcos legales de las organizaciones (Hussein, 2002).

Tomando como base el EMVS, Flora y colaboradores plantean el Marco de los Capitales de la Comunidad (MCC) (Flora et al., 2004). Los capitales son recursos o activos con los cuales cuenta 
una comunidad y que pueden ser usados para generar más recursos de diferentes tipos (Gutiérrez \& Siles, 2008). Existen capitales tangibles e intangibles, los primeros relacionados con recursos y reservas, los segundos referidos a derechos y acceso (Chambers \& Conway, 1991). Otros autores ordenan estos capitales en dos grupos o factores: humanos (humano, cultural, social y político) y materiales (natural, construido y financiero) (Flora et al., 2004; Gutiérrez-Montes et al., 2009).

Para fines de este trabajo se consideraron los siete tipos de capitales propuestos por Flora y colaboradores: natural, humano, social, cultural, político, físico y financiero (Flora et al., 2004).

En el ámbito de la formación de los jóvenes de las comunidades de Vuelta Larga y El Papayo, se formaron cinco equipos elegidos por los estudiantes. Los primeros ejercicios realizados consistieron en un acercamiento al territorio desde una perspectiva ecológica: identificación de factores bióticos y abióticos, interacciones, y participación del ser humano.

En un segundo momento, los estudiantes se reorganizaron en dos grupos, según la localidad, para la realización del diagnóstico de medios de vida y capitales de la comunidad en Vuelta Larga y El Papayo.

Cada participante enlistó diez medios de vida que considerara entre los más importantes de su comunidad. Seguidamente, todos los integrantes de cada equipo confrontaron sus respectivas listas para identificar los principales medios de vida de cada comunidad. Estos fueron colocados en una lista única, donde se reportó para cada uno el número de personas que lo registraron y la definición del medio de vida establecida por el equipo participante.

Se realizó sucesivamente un análisis de Fortalezas, Oportunidades, Debilidades y Amenazas (FODA), tomando como referencia los siete capitales de la comunidad. 
Recolección de elementos para la caracterización de la vulnerabilidad territorial en la cuenca medio-alta del Río Nagua, República Dominicana

Finalmente, en la comunidad de Vuelta Larga, se llevó a cabo un levantamiento de datos e informaciones, mediante entrevistas dirigidas a los núcleos familiares establecidos en el paraje. La numerosidad de la muestra fue determinada mediante la siguiente fórmula (Israel, 1992):

Donde:

$$
n=\frac{K^{2} p q N}{e^{2}(N-1)+K^{2} p q}
$$

$N$ : es la dimensión de la población (en este caso el total de las viviendas, o sea 70 );

$K$ : es la puntuación Z. Es un valor constante que depende del nivel de significancia estadística y mide el nivel de incertidumbre que puede ser tolerado. El nivel de significancia seleccionado para el estudio es $95 \%$, por ende el valor de $Z$ es 1.96 ;

$e$ : es el margen de error que puede ser tolerado. En el caso del estudio el valor elegido es $+/-15 \%$;

$p:$ es la desviación estándar, igual a 0.5 en este caso;

$q$ : es igual a $1-p$.

Aplicando los criterios descritos arriba, la numerosidad de la muestra de viviendas a cuyos representantes fue sometida la entrevista fue de 53 unidades.

\subsection{Levantamiento de parámetros meteo-climáticos}

Uno de los componentes importantes en la modelación de una cuenca hidrográfica es la descripción meteo-climática, basada en el análisis de datos levantados in situ.

Para fines de garantizar un punto de medición termopluviométrica representativo de las condiciones de la cuenca investigada, se instalaron un pluviómetro y un termómetro (figura N. ${ }^{\circ}$ ) en el punto de coordenadas (UTM WGS84) 19Q 395615 2133282, con las características detalladas a continuación: 
- Pluviómetro

Manual, construido según los estándares del U. S. Weather Bureau, con resolución de 0.01 pulgadas.

Recolecta la lluvia a través de un embudo, que canaliza el agua en un tubo interno graduado y removible.

Presenta una cámara externa para el sobrante, en caso de evento pluviométrico más abundante.

- Termómetro

Tipo Testo 176 T2.

Dotados de dos canales, rango de medición de $-100 \mathrm{a}+400{ }^{\circ} \mathrm{C}$, exactitud de \pm 0.2 (de $-100 \mathrm{a}+200{ }^{\circ} \mathrm{C}$ ) y \pm 0.3 (de +200.1 a $+400{ }^{\circ} \mathrm{C}$ ), resolución de $0.1^{\circ} \mathrm{C}$.

El termómetro es alojado en una garita estándar, donada por la Oficina Nacional de Meteorología (ONAMET).

Figura N. ${ }^{\circ} 1$

Estación termopluviométrica de Vuelta Larga: un particular de la instalación del termómetro

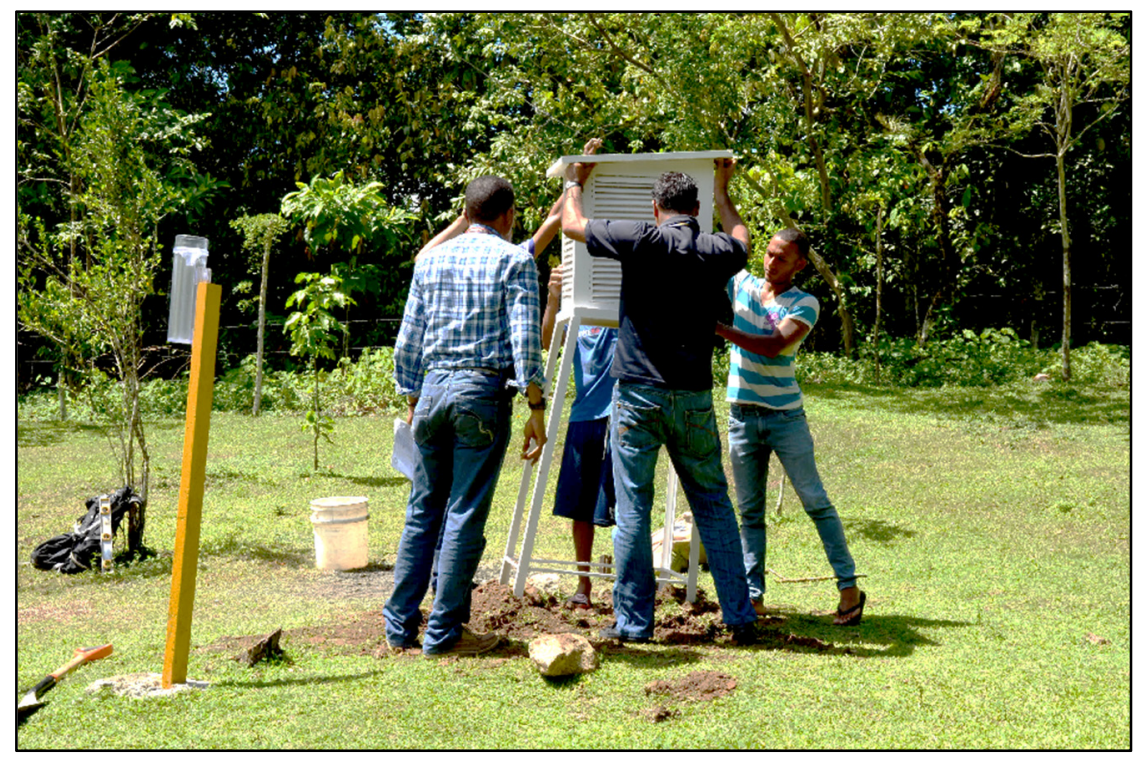

210 Ciencia y Sociedad 2016; 41(2): 201-232 
Recolección de elementos para la caracterización de la vulnerabilidad territorial en la cuenca medio-alta del Río Nagua, República Dominicana

\subsection{Medición y monitoreo de variables forestales}

Se estableció una parcela experimental de dimensiones $30 \mathrm{~m}$ x $30 \mathrm{~m}$, ubicada en Coordenadas (UTM WGS84) 19Q 394998 2134857, correspondiente al vértice suroriental, según lo especificado en la figura ${ }^{\circ} \cdot 2$.

Figura N. ${ }^{\circ} 2$

Parcela forestal de medición permanente

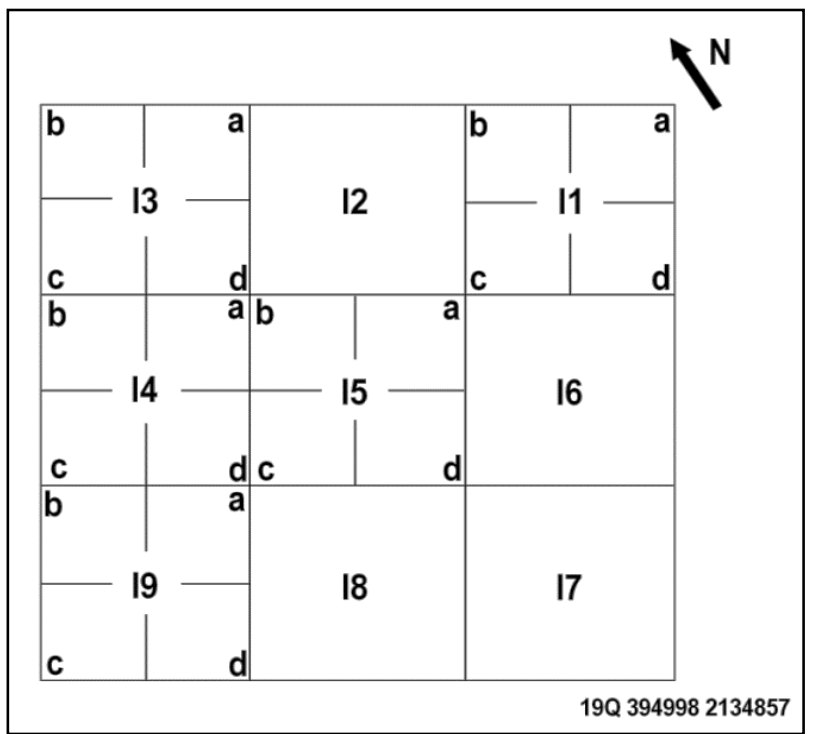

En la parcela se tomaron en cuenta las siguientes variables: localización (mediante coordenadas de uno de los vértices), altitud, exposición, clima, descripción general de bosque, identificación de las especies, mediciones dasométricas (Diámetro a la Altura del Pecho - DAP, altura, estado fitosanitario, dominancia).

Las variables dasométricas medidas son: el grosor (diámetro a la altura del pecho) y la altura de las plantas cuyo fuste tenga un diámetro superior a $10 \mathrm{~cm}$. Para los ejemplares con altura igual o superior a $1.5 \mathrm{~m}$ y con un diámetro a la altura de la base del fuste 
inferior a $10 \mathrm{~cm}$, se establecieron 4 sub-parcelas de manera aleatoria cada una de $100 \mathrm{~m}^{2}$ de superficie (Figura N. ${ }^{\circ}$ 2), donde se midieron la altura el diámetro y la altura del fuste a $10 \mathrm{~cm}$ de la base. Finalmente, para la vegetación por debajo de $1.5 \mathrm{~m}$ de altura, dentro de cada parcela se establecieron de manera aleatoria 5 sub-parcelas de $10 \mathrm{~m}^{2}$ (figura $\mathrm{N} .^{\circ}$ 2), donde se midió la altura de los ejemplares.

Las elaboraciones estadísticas fueron llevadas a cabo a través del software R (R Development Core Team, 2015), especialmente a través del paquete "Biodiversity R" (Kindt \& Coe, 2005).

\subsection{Análisis diacrónico de cobertura arbórea}

Para fines de evaluar las variaciones de cobertura arbórea ocurrida en la cuenca del Arroyo Vuelta Larga se usaron los datos del Servicio Geológico (USGS) y la Agencia Aeroespacial (NASA) de los Estados Unidos (Hansen et al., 2013). Dichos datos tienen una resolución espacial de un arco segundo (alrededor de 30 metros) y son proporcionados en forma de cierre de copas de la vegetación con altura superior a los 5 metros. En el estudio, se consideran cubiertos por árboles los pixeles que presentan un porcentaje de cierre de copas superior al 50\%. Los años de referencia para la evaluación de las variaciones son el 2000 y el 2012.

\section{Resultados}

\subsection{Descripción del área de estudio}

El área de estudio es la cuenca del Arroyo Vuelta Larga, afluente de izquierda hidrográfica en cuenca media del Río Nagua. Cuenta con una superficie de $5.69 \mathrm{~km}^{2}$ e incluye parte del territorio de la Reserva Científica Loma Guaconejo. En la zona, quedan ubicados dos poblados, constituidos por las comunidades de Vuelta Larga y El Papayo, con una población total de 842 personas. (Figura N. ${ }^{\circ}$ 3). 
Recolección de elementos para la caracterización de la vulnerabilidad territorial en la cuenca medio-alta del Río Nagua, República Dominicana

La Reserva ocupa la parte septentrional de la cuenca del Arroyo Vuelta Larga, por un total de $2.61 \mathrm{~km}^{2}$, correspondientes al $46 \%$ de su superficie.

Figura N. ${ }^{\circ} 3$

Ubicación del área de estudio

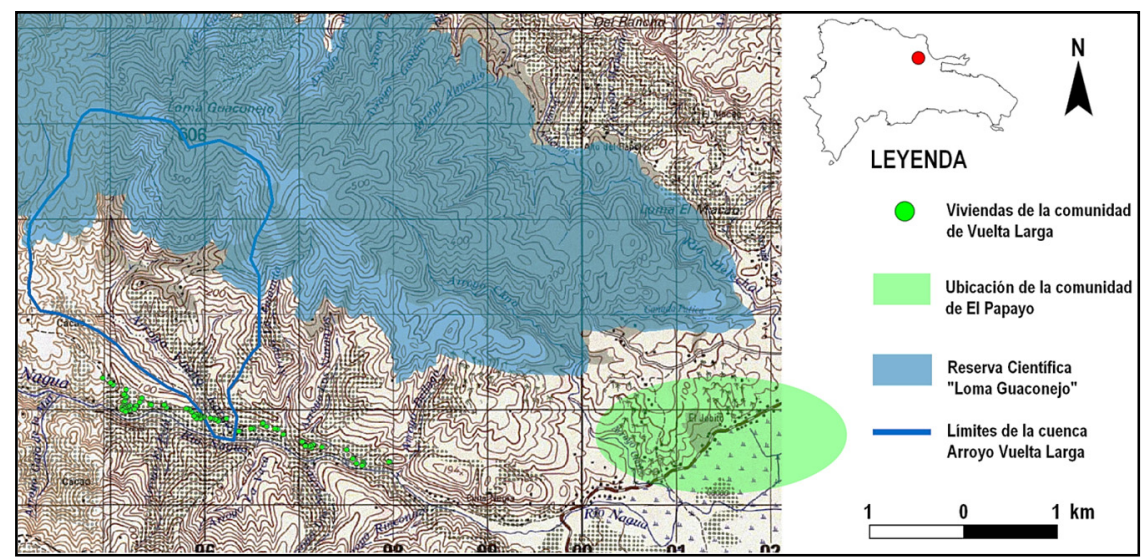

\subsection{Análisis socio-económico-ambiental}

Las evidencias recolectadas muestran que las familias de El Papayo y Vuelta Larga conviven gracias a su apego al capital natural, que permite la predominancia de actividades vinculadas al aprovechamiento del mismo.

La comunidad de El Papayo, con una mayor concentración de la población que Vuelta Larga, muestra en la definición de sus medios de vida una mayor predominancia de actividades de servicio vinculadas a procesos de urbanización.

\subsubsection{Medios de vida}

En Vuelta Larga el principal medio de vida es el cultivo del cacao, el cual es destinado prevalentemente a la venta, mientras que otros rubros son cultivados especialmente para consumo familiar. Hace 
excepción el coco, que ocupa la posición N.$^{\circ} 13$ entre los medios de vida más importantes y cuyo destino principal es la venta. (Figura N. ${ }^{\circ}$ ).

En El Papayo los principales medios de vida resultaron ser: ama de casa, producción de cacao, agricultura familiar y producción de arroz (Figura N. ${ }^{\circ}$ ).

\section{Figura N. ${ }^{\circ} 4$}

Medios de vida en las comunidades de Vuelta Larga (A) y E1 Papayo (B)

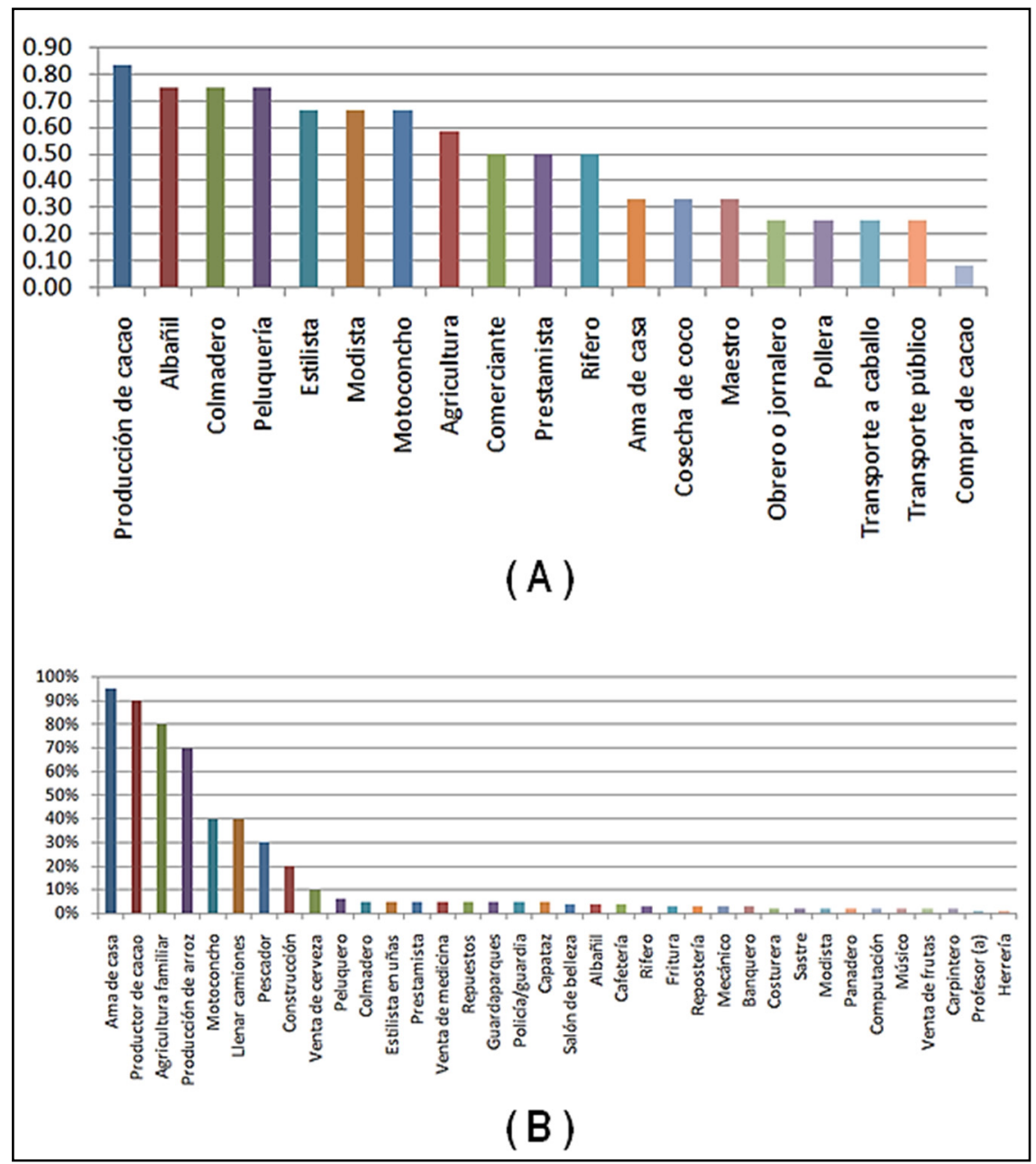


Recolección de elementos para la caracterización de la vulnerabilidad territorial en la cuenca medio-alta del Río Nagua, República Dominicana

\subsubsection{Capital natural}

Se evidencia una diferencia significativa entre los moradores de Vuelta Larga y los residentes de El Papayo, tanto en términos de conciencia ambiental como de calidad de los recursos naturales presentes en su zona de vida.

Los primeros reconocen la importancia de la calidad de los recursos naturales para que el ambiente de vida se mantenga sano. Valoran de manera particular la vegetación de la zona, que contribuye a purificar el aire, y el agua. Identifican los agroquímicos como principal fuente de contaminación, especialmente de los recursos hídricos.

Los segundos destacan que un porcentaje significativo de la población de El Papayo no está consciente de los temas ambientales y contribuye a contaminar los recursos naturales de la zona mediante manejo inadecuado de basura, materias fecales y otros elementos contaminantes. En general, respecto a los habitantes de Vuelta Larga, perciben un ambiente más contaminado, debido a las consecuencias de las actividades humanas que se llevan a cabo en el área.

\subsubsection{Capital cultural}

Ambas comunidades reconocen la raíz católica en la base de sus tradiciones y costumbres, tales como la frecuentación de la iglesia en días específicos de la semana y la celebración de eventos litúrgicos (Semana Santa, Navidad, entre otros).

Se observa un mayor apego a la vida familiar y comunitaria en Vuelta Larga, donde, de todas maneras, en los últimos años se ha destacado una menor participación en las actividades colectivas y la pérdida de algunas costumbres populares.

En El Papayo dominan actividades sociales y costumbres tales como ir al salón de belleza y salir de fiesta los fines de semana, 
tomar café como bebida social durante el día, jugar número en la lotería, jugar dominó, jugar gallos, entre otros. Se destaca la creencia en supersticiones y el consumo de alcohol. La gente es muy hospitalaria y tiene la costumbre de compartir alimentos con los vecinos.

\subsubsection{Capital humano}

En Vuelta Larga se cuentan unas 80 viviendas y una población de 350 personas, de las cuales el $95 \%$ es nativo y la restante parte es constituida por inmigrantes haitianos. Se estima que el $8 \%$ de la población es analfabeto y que un $20 \%$ es constituido por estudiantes de diferente edad.

En El Papayo hay 160 casas habitadas, con una población de 172 familias y 492 personas, de las cuales el $33 \%$ son niños y niñas.

Especialmente en Vuelta Larga, a pesar de que un 60\% de la población tiene habilidad y fuerza para el trabajo, la carencia de fuentes de empleo y estudio ha incrementado las tasas de emigración hacia zonas urbanas. Sin embargo, el porcentaje de población joven se mantiene alto, por encima del 30\%. En ella, se destaca un nivel significativo de liderazgo y capacidad de dirigir procesos comunitarios. Actualmente tienen en proceso un proyecto microhidroeléctrico y un programa formativo denominado "Universidad del Campo". Este último tiene el objetivo de contribuir a solucionar las carencias mencionadas anteriormente.

En El Papayo se observa una mayor disponibilidad de fuentes de ingreso. En la comunidad existen aproximadamente 20 profesionales en áreas de educación, medicina, y cuidado de la persona. La población ha participado en cursos sobre temas específicos, tales como introducción a la computadora, idiomas, uñas acrílicas, entre otros. 
Recolección de elementos para la caracterización de la vulnerabilidad territorial en la cuenca medio-alta del Río Nagua, República Dominicana

\subsubsection{Capital social}

Ambas comunidades cuentan con organizaciones locales.

En Vuelta Larga, operan clubes de madres, el Consejo Comunitario, la Asociación de Padres, Madres y Amigos de la Escuela, y la Asociación de Cacaocultores "Los Innovadores de Vuelta Larga" (ASOCAIVUL), principal organización comunitaria, la cual cuenta con incorporación según la Ley 122-05. La comunidad, en su proceso de desarrollo, ha venido colaborando con numerosas entidades, entre las cuales pueden citarse el Programa de Pequeños Subsidios (PPS-SGP/FMAM), Guakía Ambiente, el Instituto Dominicano de Desarrollo Integral (IDDI), la Universidad Católica Nordestana (UCNE), entre otras.

En El Papayo operan diferentes grupos de interés y organizaciones no incorporadas, entre los cuales pueden mencionarse grupos eclesiales (católicos y evangélicos), clubes de madres, grupos juveniles, consejos comunitarios, la Asociación "Los Valientes" y el Grupo "Unidad y Amor".

\subsubsection{Capital político}

En Vuelta Larga las acciones comunitarias surgen generalmente de iniciativas de las organizaciones locales, que se rigen en base a principios de solidaridad, democracia y respeto del medio ambiente. A través de ellas, se gestionan también acciones con organismos gubernamentales a diferente escala.

Miembros de la comunidad de El Papayo desempeñan el rol de representantes gubernamentales, tales como alcalde municipal y regidores. Se da importancia al cumplimiento de las leyes ambientales, especialmente aquellas relacionadas con la Reserva Científica Loma Guaconejo. Sin embargo, persisten algunos comportamientos que van en contra de la protección del ambiente. 


\subsubsection{Capital financiero}

En Vuelta Larga la producción cacaotalera es la principal actividad productiva, de la cual depende el sustento de las economías familiares. Personas que no poseen tierra trabajan como jornaleras en las labores culturales ligadas a la producción del cacao, especialmente la recolección de las mazorcas. El acceso al crédito es limitado y se vehicula principalmente a través de las empresas compradoras de los productos agrícolas, las cuales aplican tasas altas de préstamo.

En El Papayo las actividades económicas principales están ligadas a la producción de coco, arroz y cacao, así como a rifas, compraventa de productos alimenticios, bebidas alcohólicas y animales (porcinos, avícolas, vacunos). Algunas personas lucran con la venta de agregados extraídos ilegalmente del Río Nagua. También se destacan otras fuentes de ingresos menores, ligadas a provisión de servicios y/o productos. El 98\% de las familias es propietaria de tierra.

\subsubsection{Capital construido}

En Vuelta Larga, la comunidad cuenta con una escuela básica, una capilla de la iglesia católica, 80 viviendas habitadas, una carretera en condiciones muy deficientes, un sistema microhidroeléctrico (compuesto por una obra de toma, un desarenador, una casa de máquina, una línea de tubos, y una red eléctrica de distribución). Además se cuenta con secaderos y túneles para el secado del cacao. Los comunitarios cuentan con acceso al agua potable, a través del acueducto comunitario. Sin embargo, consideran deficiente el acceso a otros servicios fundamentales. Valoran mucho la posibilidad de que en el futuro, a partir del uso de la electricidad producida localmente, se establezcan microempresas que añadan valor agregado a los productos de la zona y generen empleos. 
Recolección de elementos para la caracterización de la vulnerabilidad territorial en la cuenca medio-alta del Río Nagua, República Dominicana

En El Papayo se observa una mayor urbanización, siendo evidente el mayor número de estructuras e infraestructuras presentes en su territorio. Entre ellas, se mencionan: una escuela hasta el grado de bachillerato, un molino, dos iglesias, una clínica rural, una procesadora de cacao, un play de béisbol, talleres de repuestos, casas de madera y de concreto, bancas de lotería, una farmacia, un cementerio, una carnicería, secaderos de cacao, cuarteles del Ejército Nacional, de la Policía Nacional y del Ministerio de Medio Ambiente, viveros. La población tiene acceso a diferentes servicios, tales como: teléfono, Internet, agua potable, electricidad, entre otros. Algunos de ellos no cuentan con la calidad suficiente. De manera particular, muy deficiente se considera el camino de acceso a la comunidad.

A partir de los resultados del análisis de los capitales, pudo profundizarse la situación en la comunidad de Vuelta Larga, que entre las dos presenta mayores características de aislamiento y, a la vez, mayor nivel de organización comunitaria. Entre los datos más relevantes, cabe destacar lo siguiente:

- Más del 50\% de la población usa velas como fuente principal de energía para alumbrarse.

- El manejo de la basura es inadecuado, debido a que solo el 19\% del componente orgánico se usa como abono o para alimentar a los animales, mientras que la restante parte y todo el componente inorgánico es destinado a la quema o al depósito en botaderos improvisados.

- El agua para beber no recibe ningún tratamiento en el $77 \%$ de los casos, mientras que un 11\% la hierve y otro $11 \%$ la clora.

- En promedio, el 30\% de los ingresos familiares son destinados a la alimentación, y sucesivamente a gastos de salud, transporte y educación, respectivamente. Porcentajes menores son dedicados a comunicación, entretenimiento, vivienda $\mathrm{y}$ ahorro, entre otro. 
- La fuente de energía prevalente para cocinar es la leña, aunque el 53\% de las viviendas cuenta con estufa de gas.

- La población local considera muy valiosa la posibilidad de tener acceso a diferentes tipos de electrodomésticos, una vez esté disponible la electricidad generada por la microhidroeléctrica.

- La mayor parte de las mujeres dedica la casi totalidad de su tiempo al desarrollo de las labores domésticas y las actividades ligadas a la producción de cacao.

- El 65\% de la población desea que sus hijos permanezcan en el campo, teniendo a la vez la posibilidad de seguir sus estudios.

- Las enfermedades prevalentes durante el último año fueron en orden de importancia: gripes y otras enfermedades respiratorias, hipertensión, enfermedades cardiacas, enfermedades gástricas, e infecciones renales.

\subsection{Levantamiento de parámetros meteo-climáticos}

Actualmente se cuenta con ocho meses de mediciones diarias de lluvia, mientras que todavía no se han podido levantar datos termométricos.

Los datos de lluvia levantados hasta el momento muestran que la zona está bajo la influencia de un significativo episodio seco, ligado a la ocurrencia del fenómeno de El Niño. En el período Mayo 2015 - Noviembre 2015, el déficit pluviométrico superó los $500 \mathrm{~mm}$ (Cuadro N. ${ }^{\circ} 1$ y Figura N. ${ }^{\circ}$ 5). De manera específica, cayeron $894.1 \mathrm{~mm}$, por un total 135 días con lluvia de los 214 totales y una cantidad de precipitación promedio del día lluvioso de $6.6 \mathrm{~mm}$. La lluvia máxima diaria fue de $50.8 \mathrm{~mm}$, los días $01 / 06$ y $04 / 11$. 
Recolección de elementos para la caracterización de la vulnerabilidad territorial en la cuenca medio-alta del Río Nagua, República Dominicana

\section{Cuadro N. ${ }^{\circ} 1$}

Lluvia mensual del 2015 vs lluvia total normal relativa al período 1971-2000 (Izzo et al., 2010) y diferencia correspondiente

\begin{tabular}{|c|c|c|c|}
\hline Mes & $\begin{array}{c}\text { Lluvia total } \\
\mathbf{2 0 1 5}(\mathbf{m m})\end{array}$ & $\begin{array}{c}\text { Lluvia total } \\
\text { normal }(\mathbf{m m})\end{array}$ & $\begin{array}{c}\text { Diferencia 2015 } \\
\text { normal (mm) }\end{array}$ \\
\hline Mayo & 105.9 & 244.1 & -138.2 \\
\hline Junio & 154.2 & 154.8 & -0.6 \\
\hline Julio & 103.9 & 161.7 & -57.8 \\
\hline Agosto & 102.6 & 183.3 & -80.7 \\
\hline Septiembre & 93.5 & 178.3 & -84.8 \\
\hline Octubre & 98.3 & 215.4 & -117.1 \\
\hline Noviembre & 235.7 & 266.0 & -30.3 \\
\hline
\end{tabular}

\section{Figura N. ${ }^{\circ}$}

Lluvia mensual 2015 (línea roja) vs lluvia normal (línea azul) relativa al período 1971-2000 (Izzo et al., 2010)

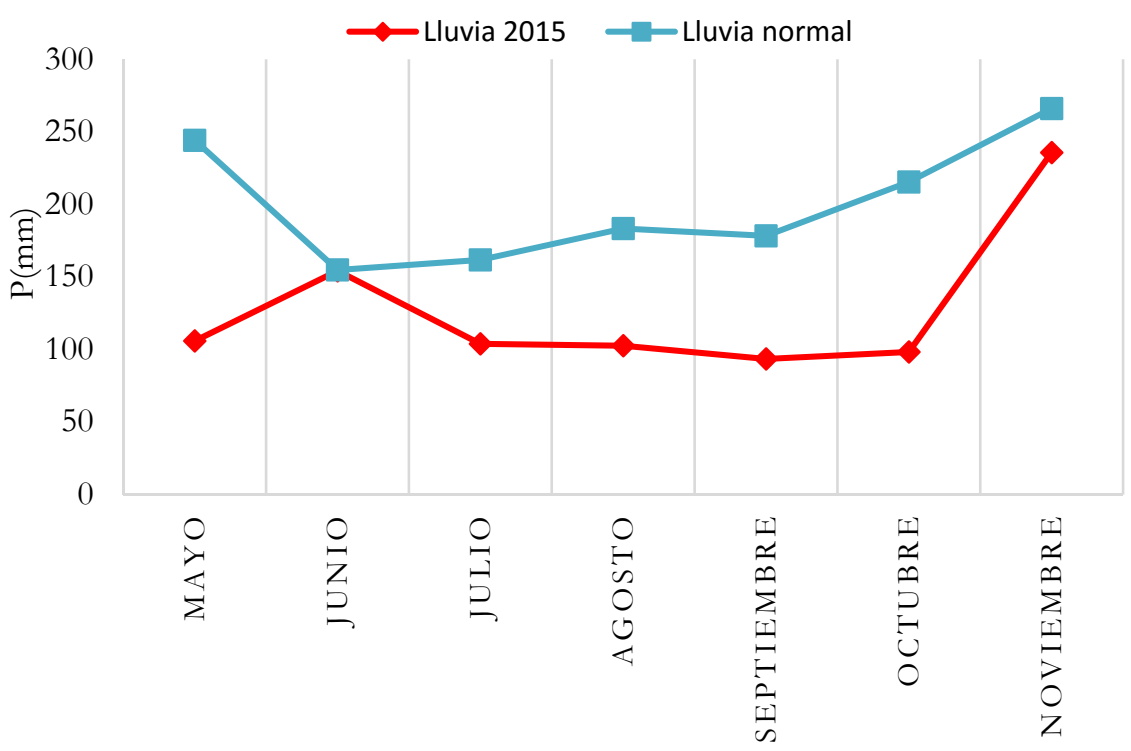


El déficit mayor se verificó en mayo y octubre, dos de los meses normalmente más húmedos. La sequía prolongada se reflejó sobre el comportamiento de las fuentes de agua de la zona, cuyo caudal bajó de manera significativa. El Arroyo Vuelta Larga, cuyo caudal de estiaje es de unos $650 \mathrm{gal} / \mathrm{min}$, bajó su flujo en los períodos más críticos a menos de $150 \mathrm{gal} / \mathrm{min}$.

Durante el desarrollo de la investigación se logró establecer un contacto significativo con la Oficina Nacional de Meteorología (ONAMET), quien donó la garita donde queda alojado el termómetro y proporcionó personal técnico que asesoró en la instalación y entrenó el personal local en los estándares a seguir para el levantamiento de los datos.

La ONAMET, junto con otras entidades, entre las cuales Guakía Ambiente, realizará visitas periódicas y llevará a cabo un control sobre los datos levantados. Luego de un período de prueba que haya demostrado que el levantamiento de las variables pluviométrica y termométrica se esté llevando a cabo según los estándares requeridos, la estación de Vuelta Larga será formalmente integrada en la red nacional de medición meteo-climática.

\subsection{Medición y monitoreo de variables forestales}

En la comunidad de Vuelta Larga queda establecida una parcela forestal de medición permanente, instalada según las características especificadas en la sección metodológica.

El proceso de medición es en fase de desarrollo, habiéndose completado de manera satisfactoria el levantamiento de los fustales. Se está completando la formación de un grupo de jóvenes de la zona, quienes en los próximos meses estarán completando el proceso de medición de línea base de la parcela, de manera que pueda darse el seguimiento requerido a lo largo del tiempo, evaluando la evolución y las dinámicas de la vegetación en ella presente. 
Recolección de elementos para la caracterización de la vulnerabilidad territorial en la cuenca medio-alta del Río Nagua, República Dominicana

En base a los resultados sobre los fustales, se obtiene la curva rango-abundancia presentada en Figura N. ${ }^{\circ}$ 6, que evidencia que la Pionía es la especie significativamente más abundante del conjunto, donde las demás especies no evidencian entre ellas diferencias significativas de abundancia.

Figura N. ${ }^{\circ} 6$

Curva rango-abundancia de la parcela forestal

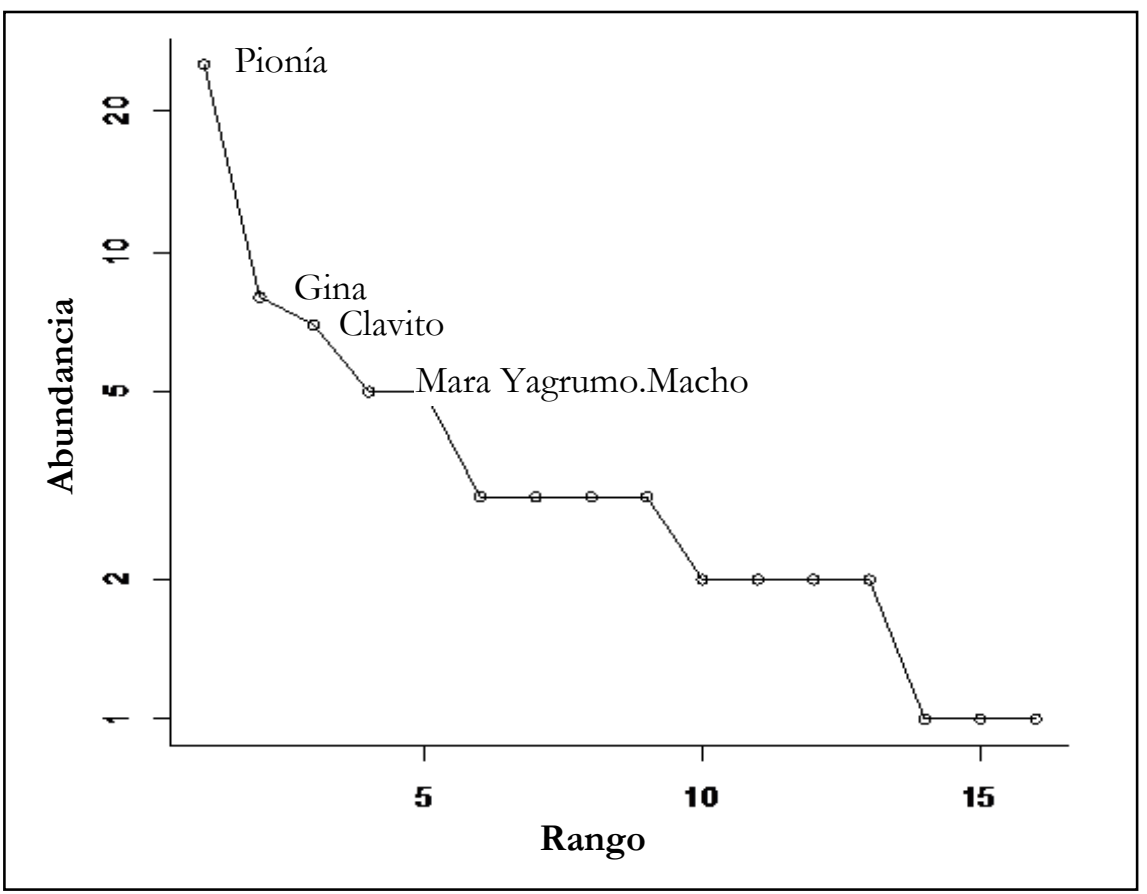

En el cuadro siguiente se presenta el número de especie presente en cada una de las sub-parcelas y en figura $\mathrm{N} .{ }^{\circ} 7$ las curvas correspondientes de diversidad según Renyi (Mayoral, 1998).

Los resultados evidencian que la curva de diversidad se mantiene por encima de uno para siete de las sub-parcelas, mientras que alcanza el valor nulo en el caso de la sub-parcela I1, donde la única especie presente es la pionía. 
Cuadro N. ${ }^{\circ} 2$

Número de especies por cada una de las sub-parcelas

\begin{tabular}{|c|c|c|c|c|c|c|c|c|}
\hline I1 & I2 & I3 & I4 & I5 & I6 & I7 & I8 & I9 \\
\hline \hline 1 & 5 & 5 & 4 & 6 & 6 & 2 & 5 & 7 \\
\hline
\end{tabular}

Figura N. ${ }^{\circ} 7$

Curva de diversidad de Renyi para cada una de las sub-parcelas

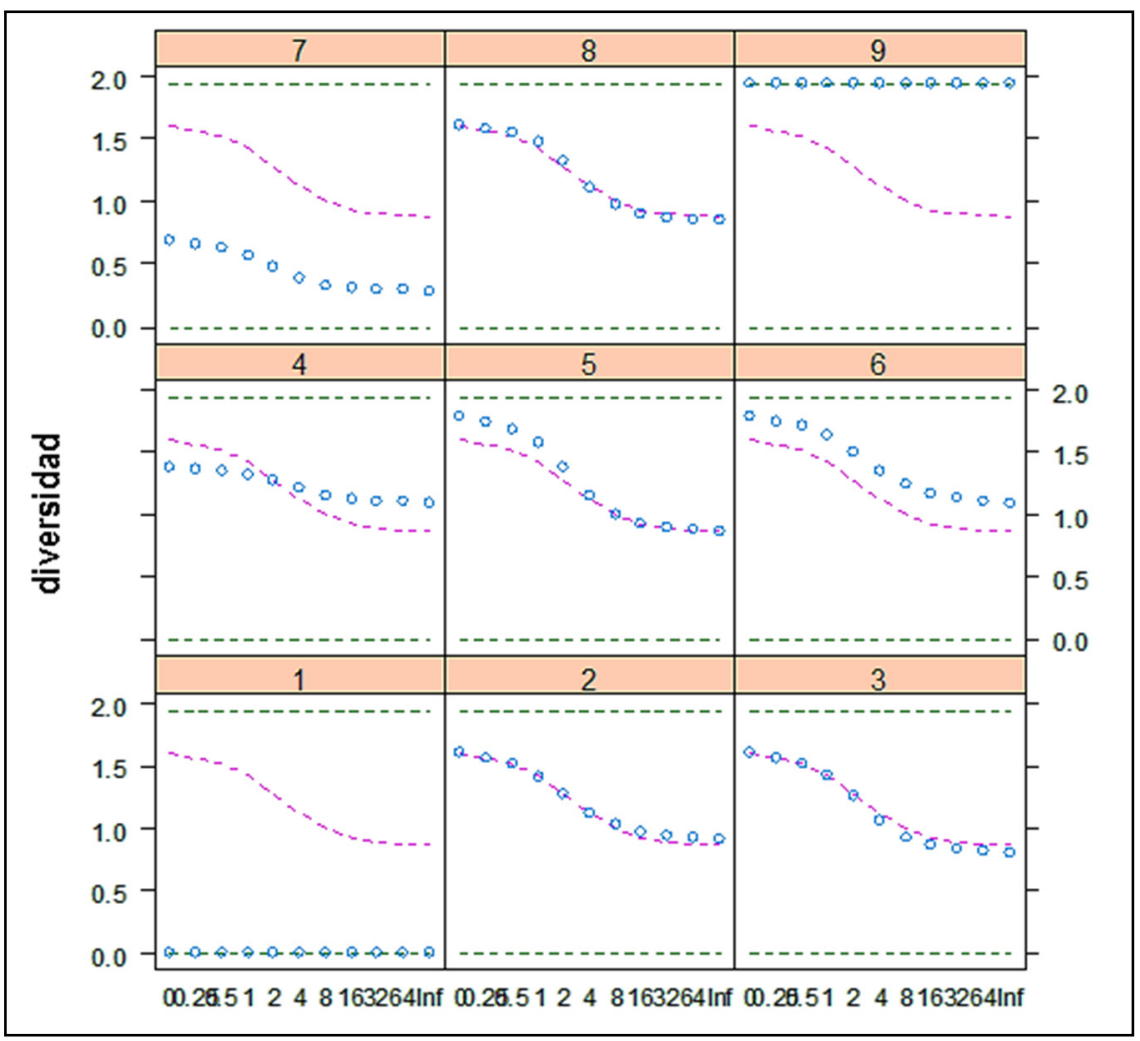

224 Ciencia y Sociedad 2016; 41(2): 201-232 
Recolección de elementos para la caracterización de la vulnerabilidad territorial en la cuenca medio-alta del Río Nagua, República Dominicana

\subsection{Análisis diacrónica de cobertura arbórea}

La cuenca del Arroyo Vuelta Larga presenta una muy buena cobertura arbórea, superior al 90\% (figura N. 8 y cuadro N. ${ }^{\circ} 3$ ).

En el período de referencia (2000-2012), se observa una ligera reducción de la cobertura arbórea, correspondiente al 2.6\% del total. Las áreas que perdieron cobertura arbórea quedan concentrada prevalentemente en las porciones central y meridional de la cuenca, en las áreas más cercanas al poblado de la comunidad de Vuelta Larga. Dichas reducciones deben atribuirse prevalentemente a cambios de uso de suelo relacionados con la instalación de parcelas de productos menores y a renovación de cacaotales.

\section{Figura $\mathbf{N}^{\circ} 8$}

Estado de cobertura arbórea en la cuenca del Arroyo Vuelta Larga (años de referencia 2000 y 2012)

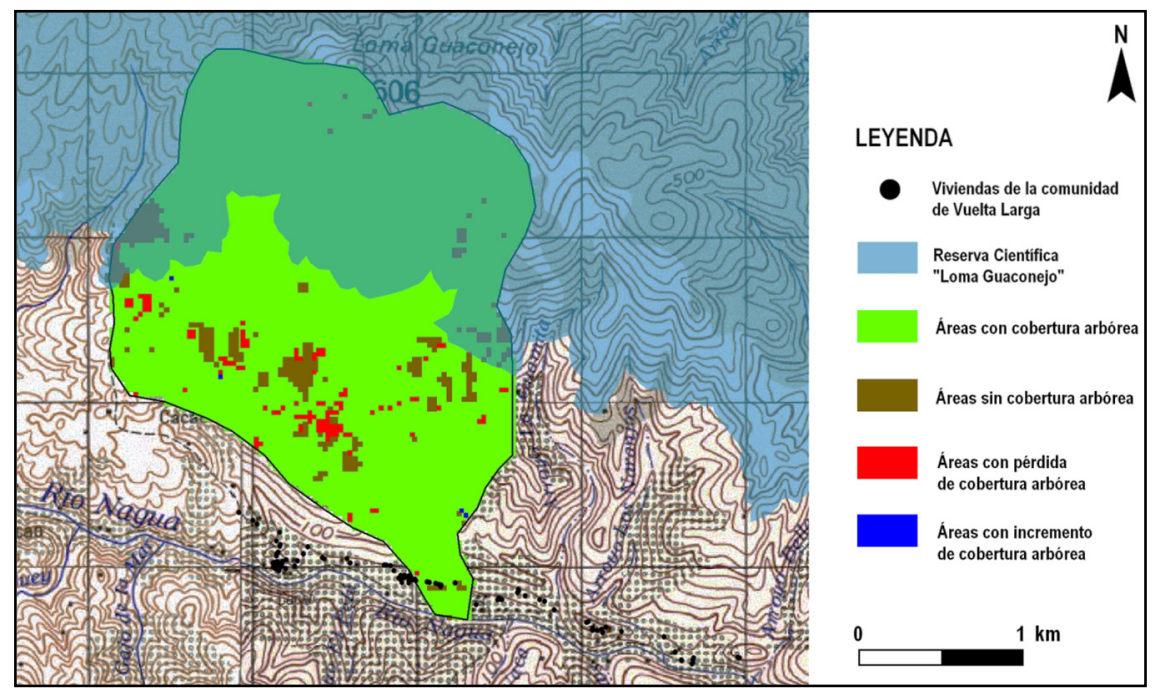


La cobertura arbórea en la porción de la cuenca ocupada por la Reserva Científica Loma Guaconejo se ha mantenido básicamente estable, con una pérdida mínima, calculable en $0.1 \%$ de la superficie, lo que permite concluir que existe un buen nivel de protección, ligado especialmente a que la población local ha asumido la importancia del área protegida y está involucrada en su conservación.

\section{Cuadro N. ${ }^{\circ} 3$}

Evoluciones de cobertura arbórea en la cuenca

\begin{tabular}{|l|c|}
\hline Superficie de la cuenca $\left(\mathrm{km}^{2}\right)$ & 5.69 \\
\hline $\begin{array}{l}\text { Superficie de la cuenca con árboles en el año } 2000 \\
\left(\mathrm{~km}^{2}\right)\end{array}$ & 5.38 \\
\hline Superficie de la cuenca con árboles en el año $2000(\%)$ & $94.5 \%$ \\
\hline $\begin{array}{l}\text { Superficie del área protegida con árboles en el año } \\
2000(\%)\end{array}$ & $95.5 \%$ \\
\hline $\begin{array}{l}\text { Superficie de la cuenca con incremento de cobertura } \\
\text { arbórea en el período 2000-2012 (\%) }\end{array}$ & $0.1 \%$ \\
\hline $\begin{array}{l}\text { Superficie del área protegida con incremento de co- } \\
\text { bertura arbórea en el período 2000-2012 (\%) }\end{array}$ & $0.0 \%$ \\
\hline $\begin{array}{l}\text { Superficie de la cuenca con pérdida de cobertura arbó- } \\
\text { rea en el período 2000-2012 (\%) }\end{array}$ & $2.6 \%$ \\
\hline $\begin{array}{l}\text { Superficie del área protegida con pérdida de cobertura } \\
\text { arbórea en el período 2000-2012 (\%) }\end{array}$ & $0.1 \%$ \\
\hline
\end{tabular}

\section{Conclusiones}

Las cuencas constituyen un sistema físico con fronteras generalmente bien definidas, caracterizado por elevada complejidad de las dinámicas que se establecen entre los diferentes componentes operantes, naturales así como antrópicos. 
Recolección de elementos para la caracterización de la vulnerabilidad territorial en la cuenca medio-alta del Río Nagua, República Dominicana

Los resultados alcanzados hasta el momento permiten identificar diferentes procesos y niveles de interacción de la población local con su territorio. De manera específica, puede observarse una progresiva urbanización de la población pasando de áreas de cuenca alta a áreas de cuenca baja, donde aumenta el nivel de individualismo y se debilita la capacidad de llevar a cabo procesos comunitarios que involucren el conjunto de la población.

A diferente nivel de urbanización se asocia también un diverso nivel de cuidado ambiental, siendo mayor la conciencia ambiental en las poblaciones de la cuenca alta. La exigencia de un mayor cuidado ambiental está surgiendo entre las nuevas generaciones de ambas comunidades.

Los principales medios de vida están vinculados al aprovechamiento de la tierra, sobre todo en sistemas agroforestales, así como a la elaboración y venta de subproductos basados en la agricultura. El crecimiento poblacional y su concentración, sobre todo en El Papayo, han permitido el surgimiento de diversos medios de vida en el sector de servicios que complementan las diversas actividades vinculadas con la agricultura ampliada. Sin embargo, son todavía escasas las fuentes de generación de empleo, especialmente en la comunidad de Vuelta Larga.

El Enfoque de Medios de Vida Sostenibles y el Marco de los Capitales de la Comunidad han resultado herramientas adecuadas para identificar los factores que pueden determinar el éxito de iniciativas de desarrollo de la comunidad, así como los puntos críticos a tomar en cuenta en estrategias de acompañamiento. Las fortalezas y oportunidades pueden potenciarse y aprovecharse para encarar el futuro y superar las debilidades, enfrentando las amenazas en un contexto de vulnerabilidad tanto en aspectos socio-económicos como ambientales.

Los niveles de cobertura arbórea son muy buenos en la cuenca del Arroyo Vuelta Larga, donde se observa una substancial estabilidad de la misma en un período de doce años (2000-2012). 
Fundamental es el levantamiento de variables meteorológicas en puntos ubicados dentro de las cuencas investigadas, para fines de poner en relación su comportamiento con aquel de otras variables significativas, especialmente los caudales de las fuentes de agua. Los datos de lluvia levantados en el período mayo-noviembre de 2015 evidencian que la zona está experimentando un profundo déficit pluviométrico, efecto de la influencia de El Niño en el área del Caribe.

Los resultados alcanzados hasta el momento constituyen una base importante para avanzar en la caracterización de la vulnerabilidad de las cuencas hidrográficas, contribuyendo a llenar un vacío de conocimiento existente en el país.

La investigación que se está llevando a cabo en la cuenca del Arroyo Vuelta Larga destaca la importancia de contar con un enfoque multidisciplinario para poder caracterizar y modelar el sistema. Los resultados obtenidos evidencian que, para fines de garantizar la continuidad que requieren las mediciones, es sumamente exitoso el involucramiento de la población local, especialmente los jóvenes, acompañando las acciones con un programa educativo orientado al fortalecimiento de sus conocimientos y el desarrollo de su capacidad investigativa.

Perspectivas futuras de investigación en la zona incluyen: producción de mapa de uso del suelo mediante imágenes satelitales de alta resolución; análisis diacrónico del uso del suelo; realización de estudios de caracterización biológicas, especialmente de la fauna acuática, orientada a la identificación de especies indicadoras de la calidad ambiental; georeferenciación de los individuos arbóreos identificados en la parcela forestal de medición permanente; estudios de suelo, con especial enfoque en la parcela forestal de medición permanente; análisis de tendencias termométricas y pluviométricas; modelación hidrológica; modelación de la cuenca hidrográfica; extensión de los estudios a la cuenca del Río Nagua; entre otras. 
Recolección de elementos para la caracterización de la vulnerabilidad territorial en la cuenca medio-alta del Río Nagua, República Dominicana

\section{Referencias}

Bolay, E. (1997). The Dominican Republic: a country between rain forest and desert. Contributions to the ecology of a Caribbean island. Bonn: Joseph Margraf Verlag.

Chambers, R. \& Conway, G. R. (1991). Sustainable rural livelihoods: practical concepts for the 21 st century. (IDS Discussion Paper, 296, 29).

Core Development Team, R. (2015). R: A Language and Environment for Statistical Computing. R Foundation for Statistical Computing. Vienna, Austria. Recuperado de http://www.R-project.org/

Flora, C. (2011). The Community Capitals Framework: a systemic approach to environmental leadership. (6, 10-11 octubre 2011, Annual Community Capitals Framework Institute. Des Moines, Iowa, USA).

Flora, C. B., Flora, J. L., Fey, S. (2004). Rural communities: legacy and change. Oxford: Westview Press.

Gutiérrez, I. \& Siles, J. (2008). Diagnóstico de medios de vida y capitales de la comunidad de Humedales de Medio Queso, Los Chiles, Costa Rica. San José: Oficina Regional para Mesoamérica y la Iniciativa CARIBE.

Gutiérrez-Montes, I., Siles, J., Bartol, P., Imbach, A. C. (2009). Merging a Landscape Management Planning Approach with the Community Capitals Framework: empowering local groups in land management processes in Bocas del Toro, Panama. Community Development, 40(2), 220-230.

Hansen, M. C., Potapov, P. V., Moore, R., Hancher, M., Turubanova, S. A., Tyukavina, A., Thau, D., Stehman, S. V., Goetz, S. J., Loveland, T. R., Kommareddy, A., Egorov, A., Chini, L., Justice, C. O., Townshend, J. R. G. (2013). High-resolution global maps of 21st-century forest cover change. Science, 342, 850-53. 
Huggins, A. E., Keel, S., Kramer, P., Núñez, F., Schill, S., Jeo, R., Chatwin, A., Thurlow, K., McPherson, M., Libby, M., Tingey, R., Palmer, M., Seybert, R. (2007). Biodiversity conservation assessment of the insular Caribbean using the Caribbean Decision Support System. [Technical Report]. Santo Domingo: The Nature Conservancy.

Hussein, K. (2002) Livelihood approaches compared: a multi-agency review of current practice. London: Omar Sattaur.

Intergovernmental Panel on Climate Change. (2007) Climate change 2007: impacts, adaptation and vulnerability. Contribution of Working Group II to the Fourth Assessment Report of the Intergovernmental Panel on Climate Change [Parry, M.L., Canziani, O.F., Palutikof, J.P., van der Linden, P.J., Hanson, C.E. (Eds.)]. Cambridge: Cambridge University Press.

Intergovernmental Panel on Climate Change. (2013) Climate Change 2013: The Physical Science Basis. Contribution of Working Group I to the Fifth Assessment Report of the Intergovernmental Panel on Climate Change [Stocker, T. F., Qin, D., Plattner, G.-K., Tignor, M., Allen, S. K., Boschung, J., Nauels, A., Xia, Y., Bex, V., Midgley, P. M. (Eds.)]. Cambridge: Cambridge University Press.

Israel, G. D. (1992). Determining Sample Siz̨e. Program Evaluation and Organizational Development, IFAS, University of Florida. PEOD-6.

Izzo, M., Aucelli, P. P. C., Maratea, A., Méndez, R., Pérez, C., Rosskopf, C. M., Segura, H. (2010). A new climatic map of the Dominican Republic based on the Thornthwaite classification. Physical Geography, 32(5), 455-472.

Izzo, M. (2011). Analisi del clima e le dinamiche climatiche nella Repubblica Dominicana e delle relative influenze sul territorio. (Tesis de doctorado), University of Molise. 
Recolección de elementos para la caracterización de la vulnerabilidad territorial en la cuenca medio-alta del Río Nagua, República Dominicana

Izzo, M. (2012a). Análisis de la vulnerabilidad del cultivo del café, con énfasis en los productores del Clúster de Café de Jarabacoa. En Análisis vulnerabilidad y plan de adaptación al cambio climático en clústeres seleccionados. Santo Domingo: Agencia de los Estados Unidos para el Desarrollo Internacional (USAID).

Izzo, M. (2012b). Análisis de la vulnerabilidad del cultivo del banano, con énfasis en los productores del Clúster de Banano. In: Análisis Vulnerabilidad y Plan de Adaptación al Cambio climático en clústeres seleccionados. Santo Domingo: Agencia de los Estados Unidos para el Desarrollo Internacional (USAID).

Izzo, M., Rathe, L., Arias Rodríguez, D. (2012). Puntos críticos para la vulnerabilidad a la variabilidad y cambio climático en la República Dominicana y su adaptación al mismo. Santo Domingo: Programa para la Protección Ambiental (USAID-TNC), Instituto Dominicano de Desarrollo Integral (IDDI).

Izzo, M., Araujo, N., Aucelli, P. P. C., Maratea, A., Sánchez, A. (2013). Land sensitivity to desertification in the Dominican Republic: an adaptation of the ESA methodology. Land Degradation and Development, 24(5), 486-498. doi: 10.1002/ ldr.2241

Kindt, R. \& Coe, R. (2005). Tree diversity analysis. A manual and software for common statistical methods for ecological and biodiversity studies. Nairobi: World Agroforestry Centre (ICRAF).

Mayoral, M. M. (1998). Renyi's entropy as an index of diversity in simple-stage cluster sampling. Information Sciences, 105(1-4), 101-114.

Pérez, C. R. \& Jury, M. R. (2012). Spatial and temporal analysis of climate change in Hispañola. Theoretical and Applied Climatology, Online First. doi 10.1007/s00704-012-0781-0 
Secretaría de Estado de Medio Ambiente y Recursos Naturales. (2004). Primera comunicación nacional. Convención Marco de las Naciones Unidas sobre Cambio Climático. Santo Domingo: Secretaría de Estado de Medio Ambiente y Recursos Naturales.

Secretaría de Estado de Medio Ambiente y Recursos Naturales. (2009). Segunda comunicación Nacional a la CMNUCC, Proyecto de Cambio Climático de la República Dominicana, 2009. Santo Domingo: el autor.

\section{Michela Izzo}

Es licenciada en Ciencias Ambientales (Universidad de Molise, Italia). Tiene una maestría en Ingeniería del Viento (Politécnico de Milán, Italia) y un doctorado en Ambiente y Territorio (Universidad de Molise, Italia). Actualmente se desempeña como encargada de Energía Renovable en el Programa de Pequeños Subsidios (PPS-SGP/FMAM) y directora Ejecutiva de Guakia Ambiente, a través del cual apoya las iniciativas de desarrollo local basadas en el empoderamiento de los grupos comunitarios. Es consultora ambiental para la realización de estudios en tema de vulnerabilidad territorial frente al cambio climático. Cuenta con más de diez publicaciones en revistas científicas internacionales sobre temas relacionados con clima y vulnerabilidad territorial.

Email: michela.izzo@guakiambiente.org michela.izzo@gmail.com

Recibido: 18-01-2016 Aprobado: 14-03-2016 